\title{
Using the NIH symptom science model to understand fatigue and mitochondrial bioenergetics
}

Qiuhua Shen, Diane Mahoney, Jill Peltzer, Faith Rahman*, Kathryn J. Krueger, John B. Hiebert and Janet D. Pierce

*Correspondence: frahman2@kumc.edu

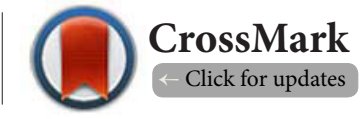

University of Kansas Medical Center, Kansas City, Kansas, 66160, United States of America.

\begin{abstract}
The symptom of fatigue is prevalent among patients with chronic diseases and conditions such as congestive heart failure and cancer. It has a significant debilitating impact on patients' physical health, quality of life, and well-being. Early detection and appropriate assessment of fatigue is essential for diagnosing, treating, and monitoring disease progression. However, it is often challenging to manage the symptom of fatigue without first investigating the underlying biological mechanisms. In this narrative review, we conceptualize the symptom of fatigue and its relationship with mitochondrial bioenergetics using the National Institute of Health Symptom Science Model (NIH-SSM). In particular, we discuss mental and physical measures to assess fatigue, the importance of adenosine triphosphate (ATP) in cellular and organ functions, and how impaired ATP production contributes to fatigue. Specific methods to measure ATP are described. Recommendations are provided concerning how to integrate biological mechanisms with the symptom of fatigue for future research and clinical practice to help alleviate symptoms and improve patients' quality of life.
\end{abstract}

Keywords: Adenosine triphosphate, fatigue, PROMIS, symptom science

\section{Introduction}

Fatigue is commonly described as one's perceived lack of energy or feelings of tiredness [1]. As such, fatigue has been observed as a normal human response to increased physical exertion as well as a physical symptom of underlying disease [2]. Fatigue has been associated with several diseases including cardiovascular and respiratory disorders, cancer, neurologic conditions, diabetes, thyroid abnormalities, rheumatic diseases, psychiatric conditions, and sleep disturbance disorders [3]. Because fatigue can lead to physical debilitation and diminished quality of life for individuals, it is important to assess and manage the symptom of fatigue for early detection of disease, monitoring illness progression, and evaluation of the effectiveness of clinical treatments $[4,5]$. One common group of patients that have severe fatigue is those suffering from heart failure. Fatigue in patients with heart failure is defined as persistent tiredness and the perception of difficulty performing daily activities because of this fatigue [6].

The National Institutes of Health Symptom Science Model (NIH-SSM) was created to guide the development of precise treatment decisions when evaluating an individual symptom such as fatigue or symptom clusters. Based on this model demonstrated in Figure 1: (1) the person presents with the symptom of fatigue; (2) phenotypic data are collected based on the patient's behavioral (self-reported), biological, and clinical information; (3) biomarkers are determined; and (4) a clinical intervention can be developed and applied [7-9]. Therefore, evaluation of patients with fatigue should incorporate multifaceted measures to assist with identifying the best treatment strategies to optimize energy levels.

Bioenergetics is the branch of biochemistry that focuses on how cells transform energy including how energy is produced, stored, or consumption of adenosine triphosphate (ATP) $[10,11]$. The ability to generate energy in the mitochondria is related to the production and utilization of the molecule called ATP. ATP is produced within the mitochondria during oxidative phosphorylation [12]. In the mitochondria the metabolism of sugars are completed and approximately 30 molecules of ATP are produced for each molecule of glucose oxidized. ATP is essential for maintaining cellular functions [13]. Thus, if ATP 


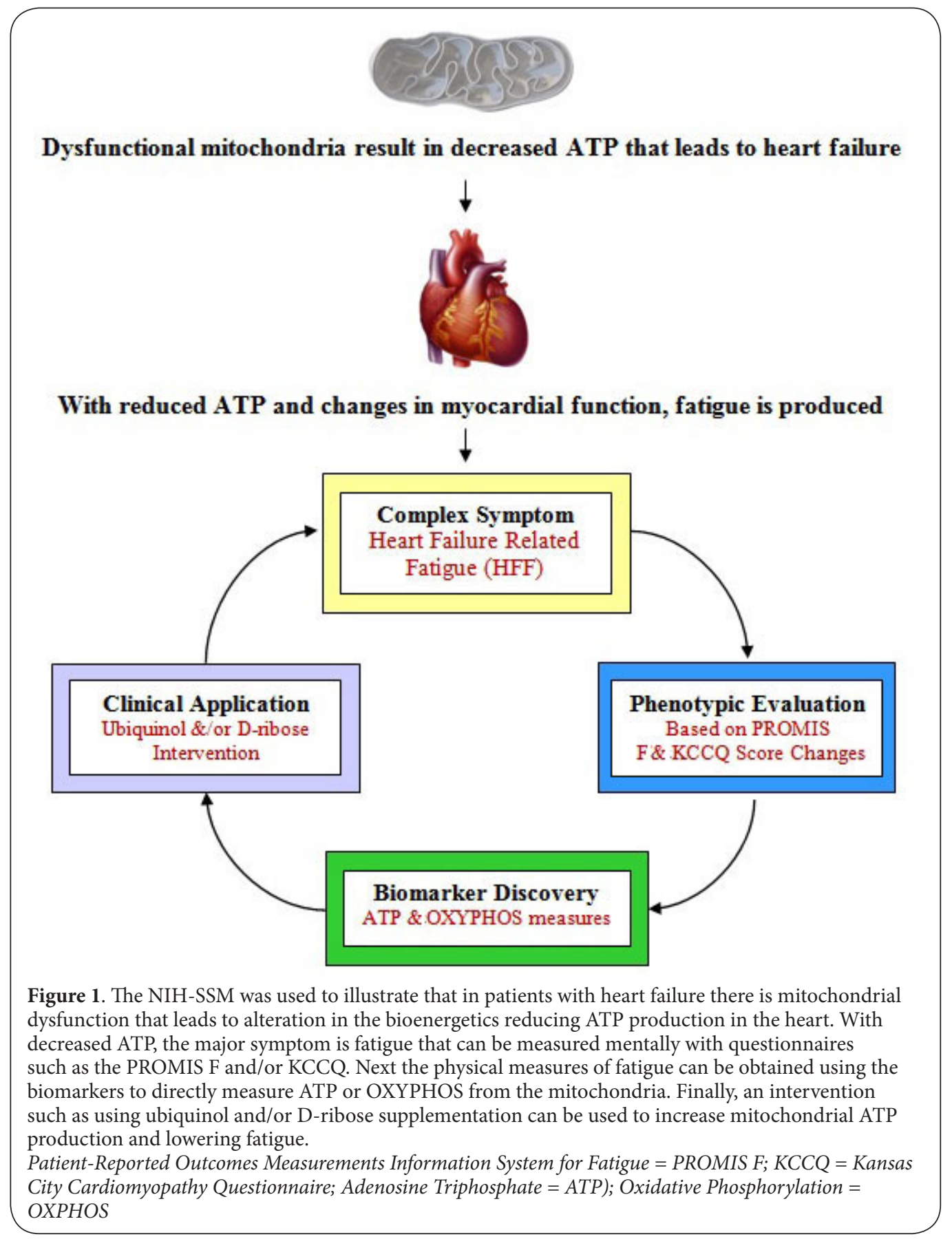

production becomes compromised, it can result in the patient experiencing fatigue. In this paper, we will be applying the $\mathrm{NIH}-\mathrm{SSM}$ to the symptom of fatigue, specifically examining the phenotypic characterization of fatigue and its relationship to mitochondrial ATP production.

This literature review is an overview of the current state of knowledge of mitochondrial bioenergetics and its relationship to fatigue. Throughout the review process, we used a synthesis matrix to identify various sources that reveal what is known, and what is not known about the phenotype of fatigue and mitochondrial performance biomarkers. We will use examples specifically related to patients with heart failure because there is a relationship between fatigue and mitochondrial dysfunction in this complex syndrome. This article will provide new and additional information concerning how to assess and examine fatigue from a mitochondrial perspective.

\section{Review}

Symptom science model

One of the four priorities of the National Institute of Nursing 
Research (NINR) is symptom science that supports the scientific foundation of nursing practice [7]. The NIH-SSM was created by scientists in the intramural research program at NINR to guide research focusing on biological mechanisms of symptoms to characterize symptom phenotypes, identify biomarkers, and develop interventions that are targeted and effective, thus advancing symptom management and care. Symptoms that occur with a single disease or more often with comorbid conditions negatively impact functionality and well-being, contributing to decreased health-related quality of life (QoL). Characterizing symptoms and identifying the biological mechanisms can lead to improved assessment and treatment to improve health, survival, and QoL. The NIH-SSM is a systematic step-by-step process that provides direction to clinicians and researchers to target symptoms that may assist with improving patient care [14]. The outline of this discussion is based upon the symptom science model (Figure 1) for fatigue and will begin with the discussion of the phenotypic evaluation of fatigue, then examine the role of bioenergetics and mitochondrial biomarkers of fatigue, and end with the clinical application of the NIH-SSM and conclusion. We will focus on mitochondrial dysfunction that has been implicated in the development of heart failure leading to the symptom of fatigue. Mitochondria in the cardiac myocytes in patients with heart failure have increased reactive oxygen species and mitochondrial damage that causes the oxidative stress and the reduction of ATP production.

\section{Complex symptom of fatigue}

A symptom is a self-reported perception of an individual's experience such as fatigue [14]. The symptom of fatigue is an important clinical concern because it is one of the most common reasons why patients seek healthcare [7]. Fatigue often impacts patient's health outcomes and QoL. Thus, assessment and management of symptoms is a hallmark of nursing practice [8]. The symptom of fatigue for clinicians and researchers can be highly subjective thus, there are multiple definitions of fatigue and many psychometric measures [15]. The ultimate goal for nurses is to reduce and improve the symptom of fatigue.

\section{Phenotypic evaluation of fatigue}

Fatigue has most often been measured by researchers and clinicians using validated self-report measures. These mental measures of fatigue are valuable for understanding patients' perspectives concerning their perceived lack of energy [4]. However, physical measures of fatigue are also important to gain a broader assessment of energy levels and assist healthcare providers with making the best treatment decisions. Physical measures of fatigue often include physical assessments and biomarkers. Serum biomarkers have been beneficial in detecting and monitoring the progression of various disease states within the general patient population [1]; thus, examining biomarkers for cellular energy could be very useful for evaluating and intervening when patients report symptoms of fatigue.

\section{Mental measures of fatigue}

Identification of the symptom of fatigue usually depends on the patient's initial self-report. It is critical to have reliable and valid tools and instruments to accurately evaluate the level of the patient's reported fatigue. Various unidimensional and multidimensional questionnaires have been developed and used to assess fatigue [16]. However, there is a major concern related to the patient's interpretation of the questionnaire items because of the subjective nature of one's perception of fatigue. The word choices used on fatigue scales may hold different meaning to patients based on their demographic backgrounds, life experiences, and disease states. Therefore, the tool or instrument used to measure fatigue should be specifically applicable to the patient populations.

Many psychometric measurements of the symptom of fatigue are available to assess self-reported fatigue focusing on mood, activity, energy, and behavior $[1,7]$. These psychosocial constructs of fatigue influence a person's perception of their health. Some of the commonly used mental measures of fatigue for the general population include the PatientReported Outcomes Measurements Information System for Fatigue (PROMIS-F) and POMS. Other mental measures of fatigue for specific patient populations include Kansas City Cardiomyopathy Questionnaire (KCCQ), Functional Assessment of Cancer Therapy: Fatigue (FACT-F), and Quality of Life in Neurological Disorders (Neuro-QoL). These measures are briefly described in Table 1.

The PROMIS is comprised of measures of mental, social, and physical health determinants across a multitude of specific domains for adults and children [17]. The PROMIS for Fatigue (PROMIS-F) item banks assess a range of self-reported symptoms, from mild subjective feelings of tiredness to an overwhelming exhaustion that may decrease a patient's ability to execute daily activities. The fatigue scale is administered as either a computer adaptive test (CAT) or a short-written form. The PROMIS fatigue instruments are available in many formats to include adults (7 tools, PROMIS-F, PROMIS F-SF 4-13a), adult cancer (1 tool, PROMIS F-Ca), pediatric self-report (4 tools, PROMIS F (v1-2), PROMIS SF(v1-2)10a) and parents serving as proxy reporters for their child (4 tools, PROMIS PPF (v1-2), PROMIS PPF-SF-F(v1-2)10a). Most of the PROMIS fatigue questionnaires rate the frequency of the test items on a 5-point Likert scale with varying combinations of experience and impact questions covering the past 7 days, except for the fatigue short form $7 \mathrm{~b}$, which includes questions about only the present day [18].

The POMS measures six distinctive mood states over the past 7 days using a 5-point Likert scale $[19,20]$. It features two scales related to perceived energy level, the Vigor factor and the Fatigue factor. The Vigor scale consists of eight questions and the Fatigue scale has seven questions. 
Shen et al, Journal of Nursing 2020,

http://www.hoajonline.com/journals/pdf/2056-9157-7-2.pdf

Table 1. List of psychometric instruments for the measurement of the symptom of fatigue.

\begin{tabular}{|c|c|c|}
\hline Name of Instrument & Number of Questions & Measurements \\
\hline PROMIS Fatigue CAT & 95 & $\begin{array}{l}\text { This tool includes a range of symptoms, from mild subjective } \\
\text { feelings of tiredness to an overwhelming, debilitating, and sustained } \\
\text { sense of exhaustion that likely decreases one's ability to execute daily } \\
\text { activities and function normally in family or social roles. }\end{array}$ \\
\hline $\begin{array}{l}\text { - } \quad \text { PROMIS Fatigue Adult Short } \\
\text { Form } 4 \mathrm{a}\end{array}$ & 4 & 3 experience and 1 impact item related to fatigue \\
\hline $\begin{array}{l}\text { - } \quad \text { PROMIS Fatigue Adult Short } \\
\text { Form } 6 \mathrm{a}\end{array}$ & 6 & 3 experience and 3 impact items related to fatigue \\
\hline $\begin{array}{l}\text { - } \quad \text { PROMIS Fatigue Adult Short } \\
\text { Form } 7 \mathrm{a}\end{array}$ & 7 & 3 experience and 4 impact items related to fatigue \\
\hline $\begin{array}{l}\text { - } \quad \text { PROMIS Fatigue Adult Short } \\
\text { Form } 7 \mathrm{~b}\end{array}$ & 7 & $\begin{array}{l}\text { Modified PROMIS Fatigue Adult Shortv1.0-Fatigue 7a to evaluate } \\
\text { daily fatigue }\end{array}$ \\
\hline $\begin{array}{l}\text { - } \quad \text { PROMIS Fatigue Adult Short } \\
\text { Form } 8 \mathrm{a}\end{array}$ & 8 & 4 experience and 4 impact items related to fatigue \\
\hline $\begin{array}{l}\text { - } \quad \text { PROMIS Fatigue Adult Short } \\
\text { Form } 13 \mathrm{a}\end{array}$ & 13 & $\begin{array}{l}6 \text { experience and } 7 \text { impact items related to fatigue to evaluate daily } \\
\text { fatigue. }\end{array}$ \\
\hline $\begin{array}{l}\text { - } \quad \text { PROMIS Fatigue Adult } \\
\text { Cancer }\end{array}$ & 54 & $\begin{array}{l}\text { All of which are also in the PROMIS Fatigue item bank }{ }^{\star} \text { but pertain } \\
\text { to cancer-related fatigue. }\end{array}$ \\
\hline FACT-F & 41 & $\begin{array}{l}\text { Assessing fatigue and anemia-related concerns in people with can- } \\
\text { cer. } 5 \text { domains. }\end{array}$ \\
\hline POMS Vigor-Activity & 8 & Mood of vigorousness, ebullience, and high energy \\
\hline POMS Fatigue-Inertia & 7 & Mood of weariness, inertia, and low energy level \\
\hline KCCQ & 15 & $\begin{array}{l}\text { Physical limitations, social interference, quality of life related to } \\
\text { fatigue in patients with heart failure }\end{array}$ \\
\hline
\end{tabular}

PROMIS, Patient-Reported Outcomes Measurements Information System; POMS, Profile of Mood States; KCCQ, Kansas City Cardiomyopathy Questionnaire;

${ }^{*}$ The experience bank contained items measuring intensity, frequency, or duration of fatigue, whereas the impact bank contained items measuring impact on physical, emotional, or social function.

Disease-specific psychometric instruments are customized for a defined subset of the population. Examples of these measurements include the KCCQ, FACT-F, and Neuro-QoL. The KCCQ is a disease-specific assessment of health status for individuals diagnosed with heart failure. The KCCQ form includes 15 questions asking about the past 2 weeks to measure an individual's physical limitations, social interference, and quality of life based on the severity of heart failure symptoms including fatigue, dyspnea, and edema [21]. The FACT-F questionnaire includes 41 questions that assess fatigue and anemia-related concerns in the domains of fatigue, physical health, social and family well-being, emotional health, and functional well-being for those with cancer [22-24]. The NeuroQoL questionnaire evaluates the physical, social, mental, and functional activities of particular symptoms, behaviors, and feelings as they relate to those with neurological disorders [25]. The Neuro-QoL includes a short and long fatigue form with 8 and 19 questions each, respectively.

\section{Physical measures of fatigue}

In addition to the mental measures of fatigue, it is important to use physical methods to evaluate the patient's level of fatigue. Such physical measures often involve exercise that can induce the patient to experience fatigue caused by the underlying biologic mechanisms related to specific diseases such as heart failure. The cardiac exercise stress test, maximal handgrip strength, and the 6-minute walk test are examples of these measures.

During a cardiac exercise stress test, the patient walks on a treadmill or cycles to increase the heart rate and make the heart work progressively harder $[26,27]$. Normal vital signs are monitored, and the symptoms of fatigue and chest discomfort are assessed. If there are signs of abnormalities in the electrocardiogram, heart rate, or blood pressure or signs of worsening fatigue or chest pain, this may indicate coronary artery disease [28]. During this type of stress test, fatigue is measured physiologically and classified in three ways: (1) peripheral fatigue; (2) central fatigue; or (3) neuromuscular fatigue [29]. Fatigue is measured at rest, during exercise, and after exercise [30]. However, the criterion used to end a stress test is often the patient's expression of the symptoms of fatigue, dyspnea, leg fatigue, or chest pain [31]. Fatigue symptoms are often recorded at each stage of the exercise stress test using the 11-point Borg scale [30,32]. This scale 
ranges from 0 to 10 with 0 being "Nothing at all" to 10 being "Very very severe (maximal)." According to Borg's psychophysiological basis for exertion, perceived fatigue is the best indicator of the degree of physical strain [33]. Often the word "fatigability" is used when describing a patient's symptom of fatigue. However, fatigability is the degree of fatigue with daily activities and differs from fatigue related to muscle endurance during an exercise stress test. A clear definition of fatigability helps standardize the measurements to objectively assess how it changes under a specified activity in terms of duration, intensity, and frequency. It is often measured during performance-based assessment [34].

Maximal handgrip strength (MHGS) can be used to examine exercise performance in healthy older subjects and in patients with chronic fatigue and other diseases. Often exercise stress tests and other maximal performances are not possible for severely tired patients. The MHGS is an alternative measure of fatigue when ergometric cycle or treadmill tests are not possible. MHGS measurement may be useful to evaluate the maximal physical performance of patients with fatigue. However, it is important to remember the sole MHGS measurement cannot replace the information provided by a maximal exercise test that can increase in exercise-induced oxidative stress $[35,36]$.

The American Thoracic Society introduced the 6-Minute Walk Test (6MWT) in 2002 to measure both aerobic capacity and endurance in walking over 6 minutes [37]. It is a simple and inexpensive test that is sensitive to fatigue-related changes when the patient walks in a 100 -foot hallway space for 6 minutes $[38,39]$. Since its inauguration, the 6 MWT has been utilized to evaluate fatigue and dyspnea in patients with various medical conditions [40,41]. At the beginning of the test, the patient stands and rates their baseline overall symptoms of fatigue and dyspnea using the Borg scale [42]. Immediately after the 6 minutes of walking or when the patient stops due to exhaustion and sits in the chair, the patient is asked to rate their symptoms of fatigue and dyspnea again using the same Borg scale [43]. The 6MWT is a useful measure of functional capacity and is helpful in measuring the patient's symptom of fatigue particularly when examining the effect of a therapeutic intervention $[44,45]$.

\section{Role of mitochondrial bioenergetics and ATP biomarkers for fatigue}

The next step of NIH-SSM after identifying the phenotypes of fatigue is identifying a biomarker to measure fatigue. Since fatigue is related to the reduction of mitochondrial ATP production, it is important to begin with understanding mitochondrial dysfunction and biomarkers to measure oxidation phosphorylation. Mitochondria are the powerhouse of cells and play a key role in maintaining cellular functions and survival by producing ATP. Dysfunction in mitochondria can cause impaired mitochondrial bioenergetics that may be due to: (1) an inadequate number of mitochondria; (2) an inability to produce the needed substrates to mitochondria; and/or (3) a problem within the mitochondrial electron transport chain and ATP machinery [12].

\section{Adenosine triphosphate production in the cell}

The ATP molecule produced within the mitochondria serves as the primary source for cellular energy. Production of ATP is required for the preservation of normal cellular function [12], and higher quantities of mitochondria can be found in cardiac muscle, brain cells, and skeletal muscles that require more ATP to function [46]. The majority of ATP is produced in the mitochondria by a process called cellular respiration (Figure 2). Cellular respiration involves several biochemical reactions to yield 36 molecules of ATP [47]. In the cell cytoplasm, glucose is metabolized by the process of glycolysis to yield 2 molecules of pyruvate, which are then shuttled into the mitochondria. The 2 molecules of pyruvate undergo oxidization to form 2 molecules of acetyl coenzyme A (acetyl CoA) to participate in the citric acid cycle. In this process, often referred as the Krebs cycle, a series of chemical reactions occur to release stored energy from proteins, carbohydrates, and fats into carbon dioxide and ATP. Glycolysis, pyruvate oxidation, and the citric acid cycle all contributes electrons through carrier molecules to the main component of ATP production, the electron transport chain (ETC). The ETC is composed of five complexes that generate approximately 32 molecules of ATP $[10,48,49]$. In the process of chemiosmosis, electrons in complexes I to IV produce an electrochemical gradient in which hydrogen ions leave the mitochondrial matrix. The electrons are then used in complex V (ATP synthase) to produce ATP by oxidative phosphorylation $[49,50]$.

\section{Impaired mitochondrial bioenergetics}

Dysfunction in cellular respiration can lead to impaired bioenergetics and a severe reduction in ATP production [51,52]. Within the complexes, ubiquinone is reduced to ubiquinol that is essential for oxidative phosphorylation. However, this process is significantly decreased with aging and in chronic diseases such heart failure. Subsequently, there is a reduction in the generation of ATP because there is less ubiquinol produced $[48,53,54]$. Impaired mitochondrial bioenergetics significantly affects cellular and organ functions, contributing to various signs and symptoms including the fatigue that patients experience. Many investigators have hypothesized that fatigue is correlated with mitochondrial dysfunction, for example, among patients with heart failure $[10,55,56]$. Reduced myocardium oxidative metabolism can lead to the inability to generate and transfer energy. This is the primary mechanism linking mitochondrial dysfunction and contractile failure. In patients with heart failure, the mitochondria cardiac myocytes have structural and energetic abnormalities leading to free radical damage that reduces ATP production. Impaired ATP production in cardiomyocytes negatively impacts the cardiac cycles, resulting in reduced cardiac output to meet the body's 


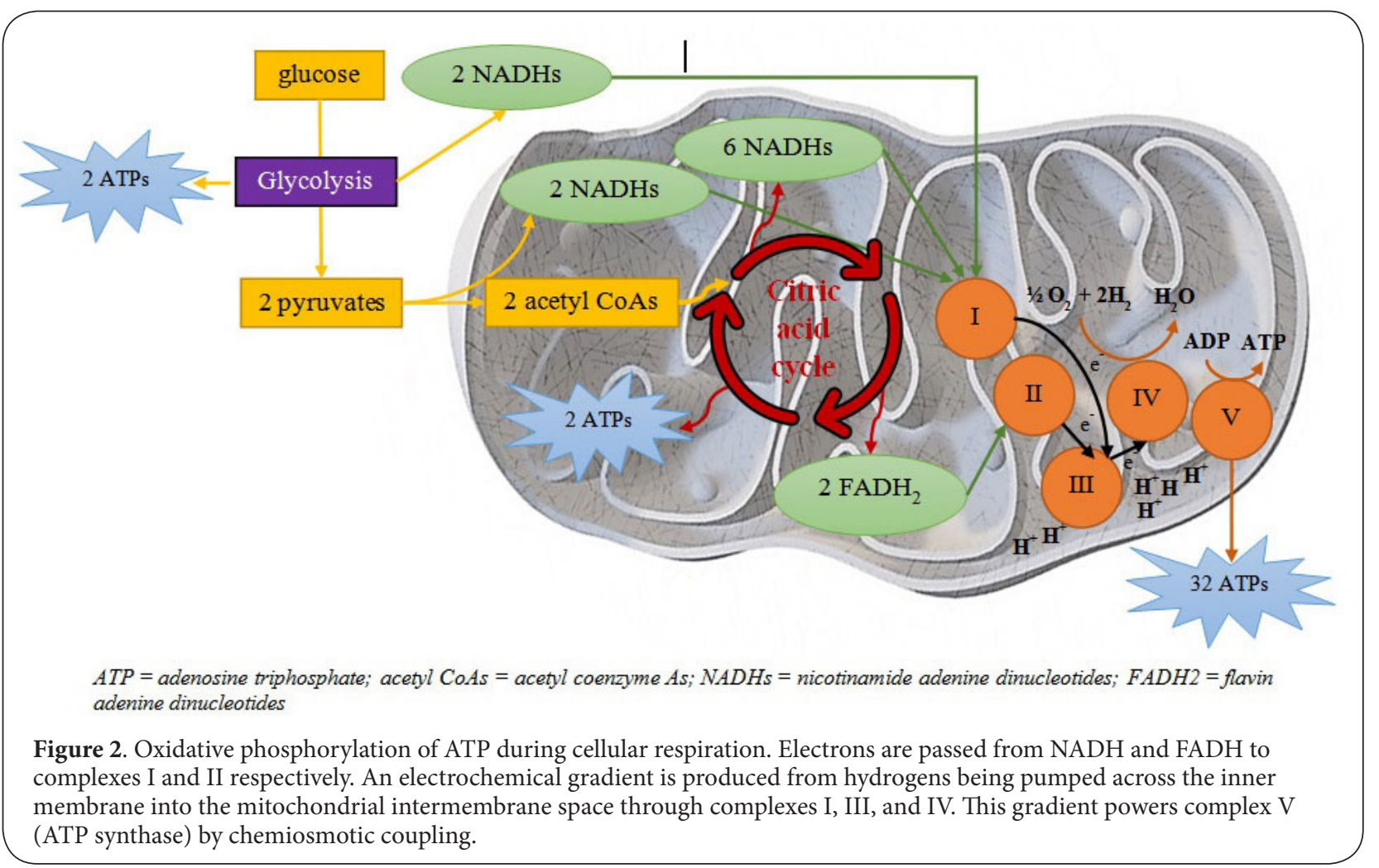

metabolic requirements. Thus, researchers are investigating whether supplemental oral ubiquinol could stimulate production of additional ATP to assist older patients with heart failure. More ATP will perhaps improve myocardial function, and the patient will experience less fatigue [57].

\section{Measuring ATP as a biomarker}

Impaired mitochondrial bioenergetics is considered one of the key underlying biological mechanisms associated with the symptom of fatigue [58]. ATP is often used by scientists as an indicator of cellular energy and is a useful biomarker to assess mitochondrial bioenergetics. The most common method for ATP measurement is using bioluminescence that relies on the enzyme luciferase's consumption of ATP. Luciferase is the substance produced in the tail of the firefly, and the oxidation of the luciferin requires ATP $[59,60]$. When luciferin and luciferase are added to a sample, an amount of light is generated that is proportional to the amount of ATP production [61]. A new and novel method to measure ATP uses atomic force microscopy (AFM) $[62,63]$ that can measure ATP at the single molecule level. AFM uses a real-time 3D-observation technique to visualize the ATP molecule in order to quantify and compare ATP molecular images. This technique still uses the firefly luciferin-luciferase method with a luminometer. There are now hand-held devices to monitor clean surfaces in hospitals based on ATP luminescence [64,65]. Recently, a point-of-contact luminometer has been adapted to measure
ATP in blood samples of patients with heart failure who are experiencing extreme fatigue [66]. There are also fluorometric and colorimetric dual-mode assays to detect ATP that uses specific fluorescent labeled aptamer to target ATP $[67,68]$. This technique allows for a very accurate quantification of ATP as well as visual colorimetric detection $[67,69]$.

\section{Measuring Electron Transport Chain (ETC) and Oxida- tive Phosphorylation (OXPHOS)}

Within the mitochondrial oxidative phosphorylation (OXPHOS) system, there are 5 complexes embedded in the mitochondrial inner membrane. These complexes assist with the aerobic synthesis of ATP. Thus, many investigators measure OXPHOS system enzymes in cells and tissues. Most commonly polarographic and spectrophotometric assays are used and can be completed in one day of work. These biomarkers often require isolation of mitochondria quickly and are very dependent of the quality of the sample. The reagents used in the biomarker must be freshly prepared and can be technically limited in resolution of direct assessment of $\mathrm{O}_{2}$ consumption and ATP production [70-72]. However, in the future more advanced development and application of these techniques may clarify the role of mitochondria on the symptom of fatigue.

\section{Clinical Application and Implications}

Fatigue is a common symptom experienced by many patients with chronic medical conditions. The severity of fatigue can 
range from mild to serious and it can have a significant impact on the patient's health as well as their cognitive, work, and safety performance. Recognizing the symptom of fatigue early by using appropriate tools and instruments such as PROMIS-F could prompt clinicians to gather additional information to facilitate an appropriate treatment plan. Careful selection of the correct psychometric fatigue tool would be an important first step when the patient reports fatigue. In addition, searching for the underlying biological mechanisms that lead to the symptom of fatigue is equally important in order to personalize therapeutic interventions. The symptom of fatigue is commonly associated with impaired ATP synthesis and an indication of mitochondrial dysfunction. Levels of ATP and/ or OXPHOS ETC enzymatic activity can be used as biomarkers for mitochondrial function and as a way to evaluate the effectiveness of interventions to treat fatigue. Researchers have developed methods to measure ATP both in the laboratory and in the clinic. These techniques are being adapted for rapid measurement of ATP using a point-of-contact luminometer. This presents an exciting opportunity for clinicians to not only assess the patient's self-reported symptom of fatigue, but also have a physiologic biomarker for fatigue.

The NIH-SSM is useful in guiding the development and implementation of studies on symptom science. For example, researchers are examining the effectiveness of dietary supplements (ubiquinol and D-ribose) in patients with heart failure with preserved ejection fraction (HFpEF) [73-76] who present with fatigue and decreased vigor [57]. In a randomized clinical trial, fatigue is measured using the Kansas City Cardiomyopathy Questionnaire (KCCQ) and vigor is measured using the Profile of Moods State (POMS) Vigor subscale. The behavioral data are combined with an additional clinical measure using a 6-minute walk test to characterize cardiac dysfunction-related fatigue. Decreased energy and physical fatigue may occur when ATP production is impaired due to mitochondrial dysfunction. ATP is measured and is used as a biomarker to assess the patient's fatigue and to evaluate the efficacy of therapeutic agents ubiquinol and D-ribose in reducing the symptom of fatigue [77].

Using the NIH-SSM to examine the symptom of fatigue has significant implications to nursing research, practice, and education. Nurse scientists are measuring mitochondrial function in relation to fatigue in a number of diseases such as heart failure and cancer $[14,57,66]$. They are using new instrumentation and technology to measure mitochondrial bioenergetics and how it relates to the symptom of fatigue in different disease processes. Findings from these studies will then be able to provide nurses in practice the evidencebased data needed to measure fatigue in the clinic or provide a treatment to reduce the symptom burden of fatigue. Finally, the NIH-SSM and mitochondrial bioenergetics as it relates to fatigue needs to be in both undergraduate and graduate nursing educational programs. Providing the pathophysiologic mechanisms for a disease is essential for nurses to understand that the symptom of fatigue has a physiologic basis.

\section{Conclusion}

The symptom of fatigue is closely related to impaired mitochondrial bioenergetics. Future research that aims to reduce the symptom burden of fatigue should focus on investigation of factors that impact this biological mechanism and on exploration of interventions that decrease fatigue and restore mitochondrial bioenergetics. Direct and indirect biomarkers for mitochondrial function are essential to evaluate the effectiveness of this type of research. Measurements of these biomarkers are often completed in laboratory and are timeconsuming and labor-intensive. Research is now focusing on the development of mitochondrial biomarkers that are simple, rapid, and easy for the clinicians to use at the bedside.

\section{Competing interests}

The authors declare that they have no competing interests.

Authors' contributions
\begin{tabular}{|l|c|c|c|c|c|c|c|}
\hline Authors' contributions & QS & DM & JP & FR & KJK & JBH & JDP \\
\hline Research concept and design & $\checkmark$ & -- & -- & $\checkmark$ & -- & $\checkmark$ & $\checkmark$ \\
\hline $\begin{array}{l}\text { Collection and/or assembly of } \\
\text { data }\end{array}$ & $\checkmark$ & -- & -- & -- & -- & -- & $\checkmark$ \\
\hline Data analysis and interpretation & $\checkmark$ & $\checkmark$ & $\checkmark$ & $\checkmark$ & $\checkmark$ & $\checkmark$ & $\checkmark$ \\
\hline Writing the article & $\checkmark$ & $\checkmark$ & $\checkmark$ & $\checkmark$ & $\checkmark$ & $\checkmark$ & $\checkmark$ \\
\hline Critical revision of the article & $\checkmark$ & $\checkmark$ & $\checkmark$ & $\checkmark$ & $\checkmark$ & $\checkmark$ & $\checkmark$ \\
\hline Final approval of article & $\checkmark$ & $\checkmark$ & $\checkmark$ & $\checkmark$ & $\checkmark$ & $\checkmark$ & $\checkmark$ \\
\hline
\end{tabular}

\section{Acknowledgement and funding}

This research was funded by the Department of Health and Human Services, National Institutes of Health, National Institute on Aging, Grant Number: 1R01AG054486-01A1. Trial registration: ClinicalTrials.gov Identifier: NCT03133793.

\section{Publication history}

Editor: David Reiss, Imperial College London, UK.

Received: 03-June-2020 Final Revised: 30-June-2020

Accepted: 14-July-2020 Published: 22-July-2020

\section{References}

1. Finsterer, J. and S.Z. Mahjoub, Fatigue in healthy and diseased individuals. Am J Hosp Palliat Care, 2014. 31(5): p. 562-75.

2. Ament, W. and G.J. Verkerke, Exercise and fatigue. Sports Med, 2009. 39(5): p. 389-422.

3. Menting, J., et al., Is fatigue a disease-specific or generic symptom in chronic medical conditions? Health Psychol, 2018. 37(6): p. 530-543.

4. Dittner, A.J., S.C. Wessely, and R.G. Brown, The assessment of fatigue: a practical guide for clinicians and researchers. J Psychosom Res, 2004. 56(2): p. 157-70.

5. Enoka, R.M. and J. Duchateau, Translating Fatigue to Human Performance. Med Sci Sports Exerc, 2016. 48(11): p. 2228-2238.

6. Evangelista, L.S., et al., Correlates of fatigue in patients with heart failure. Prog Cardiovasc Nurs, 2008. 23(1): p. 12-7.

7. Cashion, A.K., et al., National Institutes of Health Symptom Science Model sheds light on patient symptoms. Nurs Outlook, 2016. 64(5): p. 499-506.

8. Cashion, A.K. and P.A. Grady, The National Institutes of Health/National 
Institutes of Nursing Research intramural research program and the development of the National Institutes of Health Symptom Science Model. Nurs Outlook, 2015. 63(4): p. 484-7.

9. Henly, S.J., The Symptom Science Model: Challenges in Dissemination Across the Investigative Sequence. Nurs Res, 2015. 64(5): p. 329-30.

10. Sheeran, F.L. and S. Pepe, Mitochondrial Bioenergetics and Dysfunction in Failing Heart. Adv Exp Med Biol, 2017. 982: p. 65-80.

11. Kovac, L., Bioenergetics: A key to brain and mind. Commun Integr Biol, 2008. 1(1): p. 114-22.

12. Ostojic, S.M., Impaired Bioenergetics in Clinical Medicine: A Target to Tackle. Tohoku J Exp Med, 2017. 243(4): p. 227-235.

13. Yetkin-Arik, B., et al., The role of glycolysis and mitochondrial respiration in the formation and functioning of endothelial tip cells during angiogenesis. Sci Rep, 2019. 9(1): p. 12608.

14. Saligan, L.N., Collaborative Framework to Advance Symptom Science: An Intramural Perspective. J Nurs Scholarsh, 2019. 51(1): p. 17-25.

15. Lyon, D., et al., Biobehavioral examination of fatigue across populations: report from a P30 Center of Excellence. Nurs Outlook, 2014. 62(5): p. 322-31.

16. Whitehead, L., The measurement of fatigue in chronic illness: a systematic review of unidimensional and multidimensional fatigue measures. J Pain Symptom Manage, 2009. 37(1): p. 107-28.

17. Ameringer, S., et al., Psychometric Evaluation of the Patient-Reported Outcomes Measurement Information System Fatigue-Short Form Across Diverse Populations. Nurs Res, 2016. 65(4): p. 279-89.

18. Patient-Reported Outcomes Measurement Information System on Fatigue. Available from: http://www.healthmeasures.net/images/ PROMIS/manuals/PROMIS_Fatigue_Scoring_Manual.pdf.

19. Kuesten, C., J. Bi, and H.L. Meiselman, Analyzing consumers' Profile of Mood States (POMS) data using the proportional odds model (POM) for clustered or repeated observations and R package 'repolr'. Food Quality and Preference, 2017. 61: p. 38-49.

20. McNair, D.M.L., M.; Droppleman, L., EITS Manual for the Profile of Mood States. 1971, Educational and Industrial Testing Service: San Diego, California.

21. Tamartash, H.M., B.A.; Hosseini, K.; Musavi, S., The Relationship Between Disease Severity and Quality of Life in Patients With Heart Failure Based on Kansas City Questionnaire. Journal of Modern Rehabilitation, 2017. 11(3): p. 161-166.

22. Webster, K., D. Cella, and K. Yost, The Functional Assessment of Chronic IIIness Therapy (FACIT) Measurement System: properties, applications, and interpretation. Health Qual Life Outcomes, 2003. 1: p. 79.

23. Akin, S. and C. Kas Guner, Investigation of the relationship among fatigue, self-efficacy and quality of life during chemotherapy in patients with breast, lung or gastrointestinal cancer. Eur J Cancer Care (Engl), 2019. 28(1): p. e12898.

24. Al Maqbali, M., et al., Quality assessment criteria: psychometric properties of measurement tools for cancer related fatigue. Acta Oncol, 2019. 58(9): p. 1286-1297.

25. (NINDS), N.I.o.N.D.a.S., User Manual for the Quality of Life in Neurological Disorders (Neuro-QoL) Measures. 2015.

26. Charman, S.J., et al., A novel cardiac output response to stress test developed to improve diagnosis and monitoring of heart failure in primary care. ESC Heart Fail, 2018. 5(4): p. 703-712.

27. Chen, S.W., et al., A Cycling Movement Based System for Real-Time Muscle Fatigue and Cardiac Stress Monitoring and Analysis. PLoS One, 2015. 10(6): p. e0130798.

28. Tan, S.J.J., J.C. Allen, and S.Y. Tan, Determination of ideal target exercise heart rate for cardiac patients suitable for rehabilitation. Clin Cardiol, 2017. 40(11): p. 1008-1012.

29. Poole, D.C., et al., Critical Power: An Important Fatigue Threshold in Exercise Physiology. Med Sci Sports Exerc, 2016. 48(11): p. 2320-2334.

30. Weiss, K., et al., Fatigability, Exercise Intolerance, and Abnormal Skeletal Muscle Energetics in Heart Failure. Circ Heart Fail, 2017. 10(7).

31. Chrysohoou, C., et al., Cardiovascular effects of high-intensity interval aerobic training combined with strength exercise in patients with chronic heart failure. A randomized phase III clinical trial. Int J Cardiol, 2015. 179: p. 269-74.

32. Richardson, C.A., et al., Walking energetics, fatigability, and fatigue in older adults: the study of energy and aging pilot. J Gerontol A Biol Sci Med Sci, 2015. 70(4): p. 487-94.

33. Borg, G.A., Psychophysical bases of perceived exertion. Med Sci Sports Exerc, 1982. 14(5): p. 377-81.

34. Kim, I., et al., Evaluation of fatigability measurement: Integrative review. Geriatr Nurs, 2018. 39(1): p. 39-47.

35. Jammes, Y., et al., Maximal handgrip strength can predict maximal physical performance in patients with chronic fatigue. Clin Biomech (Bristol, Avon), 2020. 73: p. 162-165.

36. Strandkvist, V., et al., Hand grip strength is associated with fatigue among men with COPD: epidemiological data from northern Sweden. Physiother Theory Pract, 2020. 36(3): p. 408-416.

37. Laboratories, A.T.S.C.o.P.S.f.C.P.F., ATS statement: guidelines for the sixminute walk test. Am J Respir Crit Care Med, 2002. 166(1): p. 111-7.

38. Ferreira, J.P., et al., Clinical correlates and outcome associated with changes in 6-minute walking distance in patients with heart failure: findings from the BIOSTAT-CHF study. Eur J Heart Fail, 2019. 21(2): p. 218-226.

39. Spina, E., et al., Six-minute walk test is reliable and sensitive in detecting response to therapy in CIDP. J Neurol, 2019. 266(4): p. 860-865.

40. Witherspoon, J.W., et al., 6-minute walk test as a measure of disease progression and fatigability in a cohort of individuals with RYR1-related myopathies. Orphanet J Rare Dis, 2018. 13(1): p. 105.

41. Montes, J., et al., Six-Minute Walk Test demonstrates motor fatigue in spinal muscular atrophy. Neurology, 2010. 74(10): p. 833-8.

42. Giannitsi, S., et al., 6-minute walking test: a useful tool in the management of heart failure patients. Ther Adv Cardiovasc Dis, 2019. 13: p. 1753944719870084.

43. Gurses, H.N., et al., The relationship of sit-to-stand tests with 6-minute walk test in healthy young adults. Medicine (Baltimore), 2018. 97(1): $p$. e9489.

44. Kargarfard, M., et al., Randomized Controlled Trial to Examine the Impact of Aquatic Exercise Training on Functional Capacity, Balance, and Perceptions of Fatigue in Female Patients With Multiple Sclerosis. Arch Phys Med Rehabil, 2018. 99(2): p. 234-241.

45. Poort, H., et al., Study protocol of the TIRED study: a randomised controlled trial comparing either graded exercise therapy for severe fatigue or cognitive behaviour therapy with usual care in patients with incurable cancer. BMC Cancer, 2017. 17(1): p. 81.

46. Herbers, E., et al., Tissue specific differences in mitochondrial DNA maintenance and expression. Mitochondrion, 2019. 44: p. 85-92.

47. Mookerjee, S.A., et al., Quantifying intracellular rates of glycolytic and oxidative ATP production and consumption using extracellular flux measurements. J Biol Chem, 2017. 292(17): p. 7189-7207.

48. van der Bliek, A.M., M.M. Sedensky, and P.G. Morgan, Cell Biology of the Mitochondrion. Genetics, 2017. 207(3): p. 843-871.

49. Bonora, M., et al., ATP synthesis and storage. Purinergic Signal, 2012. 8(3): p. 343-57.

50. Neupane, P., et al., ATP Synthase: Structure, Function and Inhibition. Biomol Concepts, 2019. 10(1): p. 1-10.

51. Pieczenik, S.R. and J. Neustadt, Mitochondrial dysfunction and molecular pathways of disease. Exp Mol Pathol, 2007. 83(1): p. 84-92.

52. Tomas, C., et al., Cellular bioenergetics is impaired in patients with chronic fatigue syndrome. PLoS One, 2017. 12(10): p. e0186802.

53. Liu, S.Z. and D.J. Marcinek, Skeletal muscle bioenergetics in aging and heart failure. Heart Fail Rev, 2017. 22(2): p. 167-178.

54. Sorrentino, V., K.J. Menzies, and J. Auwerx, Repairing Mitochondrial Dysfunction in Disease. Annu Rev Pharmacol Toxicol, 2018. 58: p. 353389.

55. De Jong, K.A. and G.D. Lopaschuk, Complex Energy Metabolic Changes 
Shen et al, Journal of Nursing 2020,

in Heart Failure With Preserved Ejection Fraction and Heart Failure With Reduced Ejection Fraction. Can J Cardiol, 2017. 33(7): p. 860-871.

56. Mortensen, S.A., et al., The effect of coenzyme Q10 on morbidity and mortality in chronic heart failure: results from Q-SYMBIO: a randomized double-blind trial. JACC Heart Fail, 2014. 2(6): p. 641-9.

57. Pierce, J.D., et al., Study protocol, randomized controlled trial: reducing symptom burden in patients with heart failure with preserved ejection fraction using ubiquinol and/or D-ribose. BMC Cardiovasc Disord, 2018. 18(1): p. 57.

58. Armstrong, C.W., et al., Metabolism in Chronic Fatigue Syndrome. Advances in Clinical Chemistry, Vol 66, 2014. 66: p. 121-172.

59. Thorne, N., J. Inglese, and D.S. Auld, Illuminating insights into firefly luciferase and other bioluminescent reporters used in chemical biology. Chem Biol, 2010. 17(6): p. 646-57.

60. Rajendran, M., et al., Imaging Adenosine Triphosphate (ATP). Biol Bull, 2016. 231(1): p. 73-84.

61. Gentry, D.M., et al., Correlations Between Life-Detection Techniques and Implications for Sampling Site Selection in Planetary Analog Missions. Astrobiology, 2017. 17(10): p. 1009-1021.

62. Li, N., et al., MoS2-LA-PEI nanocomposite carrier for real-time imaging of ATP metabolism in glioma stem cells co-cultured with endothelial cells on a microfluidic system. Biosensors \& Bioelectronics, 2018. 99: p. 142-149.

63. Chada, N., et al., Single-molecule observation of nucleotide induced conformational changes in basal SecA-ATP hydrolysis. Science Advances, 2018. 4(10).

64. Ellis, O., et al., How to better monitor and clean irregular surfaces in operating rooms: Insights gained by using both ATP luminescence and RODAC assays. Am J Infect Control, 2018. 46(8): p. 906-912.

65. Mishra, P.M., K.P.; Singh, D.; Ganju, L.; Kumar, B.; Singh, S.B., Advances in Rapid Detection and Antimicrobial Susceptibility Tests. Defence Life Science Journal, 2019. 4(1): p. 12-20.

66. Pierce, J., et al., Development of a point-of-contact technique to measure adenosine triphosphate: A quality improvement study. Ann Med Surg (Lond), 2019. 41: p. 29-32.

67. Cheng, D.D., et al., Fluorescence and colorimetric detection of ATP based on a strategy of self-promoting aggregation of a water-soluble polythiophene derivative. Chemical Communications, 2015. 51(40): $p$. 8544-8546.

68. Luo, J.P., et al., Signal amplification by strand displacement in a carbon dot based fluorometric assay for ATP. Microchimica Acta, 2018. 185(8).

69. Lu, S.S., et al., Enzyme-free aptamer/AuNPs-based fluorometric and colorimetric dual-mode detection for ATP. Sensors and Actuators B-Chemical, 2018. 265: p. 67-74.

70. Barrientos, A., F. Fontanesi, and F. Diaz, Evaluation of the mitochondrial respiratory chain and oxidative phosphorylation system using polarography and spectrophotometric enzyme assays. Curr Protoc Hum Genet, 2009. Chapter 19: p. Unit19 3.

71. Stary, C.M., A high-resolution method for assessing cellular oxidative phosphorylation efficiency: bringing mitochondrial bioenergetics into focus. Focus on "Direct real-time quantification of mitochondrial oxidative phosphorylation efficiency in permeabilized skeletal muscle myofibers". Am J Physiol Cell Physiol, 2016. 311(2): p. C237-8.

72. Shin, B., et al., Mitochondrial Oxidative Phosphorylation Regulates the Fate Decision between Pathogenic Th17 and Regulatory T Cells. Cell Rep, 2020. 30(6): p. 1898-1909 e4.

73. Omran, H., et al., D-Ribose improves diastolic function and quality of life in congestive heart failure patients: a prospective feasibility study. Eur J Heart Fail, 2003. 5(5): p. 615-9.

74. Melike Bayram, D.P., JA St Cyr, and William T Abraham, Clinical Significance and Applications of D-Ribose in Cardiovascular Disease. International Archives of Cardiovascular Diseases, 2018. 2(2): p. 1-9.
75. Sharma, A., et al., Coenzyme Q10 and Heart Failure: A State-of-the-Art Review. Circ Heart Fail, 2016. 9(4): p. e002639.

76. Fukuda, S., et al., Ubiquinol-10 supplementation improves autonomic nervous function and cognitive function in chronic fatigue syndrome. Biofactors, 2016. 42(4): p. 431-40.

77. Sabbah, H.N., Targeting the Mitochondria in Heart Failure: $A$ Translational Perspective. JACC Basic TransI Sci, 2020. 5(1): p. 88-106.

\section{Citation:}

Shen Q, Mahoney D, Peltzer J, Rahman F, Krueger KJ, Hiebert JB and Pierce JD. Using the NIH symptom science model to understand fatigue and mitochondrial bioenergetics. J Nurs. 2020; 7:2. http://dx.doi.org/10.7243/2056-9157-7-2 